\title{
Valeurs propres des systèmes dynamiques définis par des substitutions de longueur variable
}

\author{
B. HOST \\ Départment de Mathématiques, CSP-Université de Paris-Nord, \\ 93430-Villetaneuse, France
}

(Received 3 July 1985 and revised 14 February 1986)

\begin{abstract}
Parmi les systèmes dynamiques définis par des substitutions, les mieux connus sont ceux qui proviennent de substitutions à longueur constante. F. M. Dekking [1] a déterminé les valeurs propres de ces systèmes, et, plus récemment, M. Queffelec a étudié leur type spectral maximal. On se propose ici de déterminer les valeurs propres de ces systèmes dans le cas général des substitutions à longueur variable; dans cette direction, un résultat partiel a été obtenu par J. C. Martin [3] dans le cas des substitutions de 2 lettres, pour les valeurs propres correspondant à des fonctions propres continues.
\end{abstract}

\section{Préliminaires et énoncé du théorème}

(1.1) Définitions. Les systèmes dynamiques définis par substitutions semblent avoir été introduits par W. H. Gottschalk [2]; on pourra aussi se référer à [4] pour les résultats essentiels utilisés ici.

Soit $\boldsymbol{A}$ un ensemble fini non vide, que l'on appellera l'alphabet, formé de $s$ éléments que l'on appellera les lettres. On note $A^{*}$ l'ensemble des suites finies non vides de lettres. Pour un élément $w$ de $A^{*},|w|$ désignera la longueur de $w$.

Soit $\zeta$ une substitution sur $A$, c'est-à-dire une application de $A$ dans $A^{*} . \zeta$ définit naturellement une application, encore notée $\zeta$, de $A^{*}$ dans $A^{*}$ : pour un élément $w=\left(w_{0} w_{1} \cdots w_{n}\right)$ de $A^{*}, \zeta(w)$ est obtenu en concaténant $\zeta\left(w_{0}\right), \zeta\left(w_{1}\right), \ldots, \zeta\left(w_{n}\right)$. On peut alors itérer la substitution: pour $k>0, \zeta^{k}$ désigne la $k$-ième itérée de $\zeta$, qui est aussi une substitution sur $A$.

Supposons de plus qu'il existe une lettre de $A$, notée 0 , telle que la première lettre de $\zeta(0)$ soit 0 . Alors, par récurrence, pour tout $k>0$ la suite $\zeta^{k+1}(0)$ est un prolongement de la suite $\zeta^{k}(0)$. Si la longueur de $\zeta^{k}(0)$ tend vers l'infini, on obtient à la limite une suite infinie $u \in A^{\mathbb{N}}$. Cette suite peut aussi être définie de la façon suivante: la substitution $\zeta$ génère, par concaténation, une application de $A^{\mathbb{N}}$ dans lui-même que l'on note encore $\zeta$. $u$ est alors l'unique élémement de $A^{\mathbb{N}}$ tel que

$$
u_{0}=0 \quad \text { et } \quad \zeta(u)=u \text {. }
$$

Munissons l'espace compact $A^{\mathbb{N}}$ du shift $T$ : pour $x=\left(x_{n}\right) \in A^{\mathbb{N}}, T x$ est défini par

$$
(T x)_{n}=x_{n+1} \quad \text { pour tout } n \geq 0 .
$$


Soit $X$ l'orbite fermée de $u$ sous le shift $T$. Le système dynamique $(X, T)$ sera appelé le système dynamique défini par la substitution $\zeta$. A priori, si on choisit une autre lettre initiale $u_{0}^{\prime}$ au lieu de 0 on obtient une autre suite $u^{\prime}$ et un autre système $\left(X^{\prime}, T\right)$; cependant, pour les substitutions primitives (cf. (1.2)) que l'on considèrera ici, le système $(X, T)$ défini par $u$ est minimal, et $u^{\prime}$ appartient à $X$, donc les systèmes dynamiques $(X, T)$ et $\left(X^{\prime}, T\right)$ sont identiques.

Certains auteurs préfèrent construire des suites bilatères, de sorte que $T$ soit un homéomorphisme de $X$; ce procédé semble inutile: pour les substitutions primitives considérées, $T$ est surjective par minimalité; d'autre part, d'après $[4$, Chap. $4, \S 1]$, l'ensemble $Y$ des points de $X$ ayant plusieurs images réciproques est fini, et $T^{-1} Y$ est fini; ainsi, l'ensemble $D=\bigcup_{k \in \mathbb{Z}} T^{k} Y$ est dénombrable, et $T$ est un homéomorphisme de $X \backslash D$.

(1.2) Les hypothèses. On supposera désormais que la substitution $\zeta$ est primitive, c'est-à dire que:

pour tout $a, b \in A$, et pour tout entier $k$ assez grand, la lettre $b$ apparaît dans $\zeta^{k}(a)$. Alors, pour toute lettre $a,\left|\zeta^{k}(a)\right|$ tend vers l'infini, et on peut bien définir la suite infinie $u$. De plus, le système $(X, T)$ est à la fois minimal et uniquement ergodique; on notera $\mu$ son unique mesure invariante.

On supposera aussi que la substitution $\zeta$ est injective sur les lettres, c'est-à-dire que, si $a$ et $b$ sont deux lettre distinctes, $\zeta(a)$ et $\zeta(b)$ sont distincts.

On aura besoin aussi d'une hypothèse de reconnaissabilité de la suite $u$. Pour $x \in A^{\mathbb{N}}$, et $m<n$ deux entiers, on notera $x\left[m, n\right.$ [ l'élément $\left(x_{m} x_{m+1} \cdots x_{n-1}\right)$ de $A^{*}$.

Soit $E$ la partie de $\mathbb{N}$ définie par

$$
E=\{0\} \cup\{\mid \zeta(u[0, p[) \mid: p>0\} .
$$

On supposera désormais que:

il existe un entier $k>0$ tel que, si $n$ appartient à $E$ et si $m$ vérifie

alors $m$ appartient à $E$.

$$
u[n, n+k[=u[m, m+k[\text {, }
$$

On dit alors que la substitution $\zeta$ est reconnaissable, et que $k$ est son indice de reconnaissabilité.

La substitution $\zeta$ étant reconnaissable, la suite $u$ ne peut pas être périodique. J. C. Martin [3] affirme qu'une substitution sur un alphabet de 2 lettres qui définit une suite non périodique est reconnaissable, mais sa démonstration n'est pas convaincante. Une condition suffisante pour que la substitution primitive $\zeta$ soit reconnaissable est qu'elle soit post-fixée, c'est-à-dire que:

$a$ et $b$ étant 2 lettres distinctes, $\zeta(a)$ n'est pas égal à la partie droite de $\zeta(b)$, c'est-à-dire $\zeta(a) \neq \zeta(b)$ et il n'existe aucun $w \in A^{*}$ tel que $\zeta(b)=w \zeta(a)$.

(1.3) Cobords. On dira que l'élément $w$ de $A^{*}$ est un mot de $u$ s'il apparaît dans $u$, c'est-à-dire s'il existe 2 entiers $m$ et $n$ avec $0 \leq m<n$ tels que $w=u[m, n[$.

Une application $h$ de $A$ dans l'ensemble des complexes de module 1 sera appelée un cobord de $\zeta \mathrm{si}$, pour tout mot $u\left[m, n\left[\right.\right.$ de $u$ vérifiant $u_{n}=u_{m}$ on a $h\left(u_{m}\right) h\left(u_{m+1}\right) \cdots h\left(u_{n-1}\right)=1$. Pour que $h$ soit un cobord, il faut et il suffit qu'il existe une application $f$ de $A$ dans l'ensemble des complexes de module 1 telle que:

pour tout mot $(a b)$ de 2 lettres de $u, f(b)=f(a) h(a)$. 
Dans les cas les plus simples, par exemple si l'alphabet $A$ est formé de 2 lettres, tout cobord de $\zeta$ est constant et égal à 1 .

(1.4) ThÉOREME. Soit $\zeta$ une substitution primitive, injective sur les lettres et reconnaissable sur un alphabet $A$, et soit $(X, T, \mu)$ le système dynamique défini par cette substitution:

(i) Toute fonction propre de $(X, T, \mu)$ est égale presque partout à une fonction propre continue.

(ii) Pour que le complexe $\lambda$ de module 1 soit une fonction propre de $(X, T, \mu)$, il faut et il suffit qu'il existe un entier $p>0$ tel que, pour toute lettre $a \in A$, la limite

$$
h(a)=\lim _{n \rightarrow+\infty} \lambda^{\left|\zeta^{p n}(a)\right|}
$$

existe, et que $h$ soit un cobord de $\zeta$.

\section{Discussion}

(2.1) L'entier $p$ intervenant dans l'énoncé du théorème peut être déterminé à priori:

Soit $J$ l'application de $\boldsymbol{A}$ dans lui-même qui à chaque lettre associe la première lettre de $\zeta(a)$. A étant fini, la suite $\left(J^{n}\right)$ des itérés de $J$ est périodique à partir d'un certain rang. La période de cette suite, qu'on peut appeler la période des initiales, peut être choisie comme entier $p$. En particulier, si pour toute lettre a la première lettre de $\zeta(a)$ est $a$, on peut prendre $p=1$. Ce résultat sera démontré au $\S 5$.

(2.2) En appliquant le théorème aux substitutions à la longueur constante, on peut retrouver le résultant de [1].

Supposons que la substitution $\zeta$ est de longueur constante $q$. Pour tout $n$ et toute lettre $a,\left|\zeta^{n}(a)\right|=q^{n}$. Soient $t \in \mathbb{R}$ et $\lambda=e^{2 \pi i t}$. Pour que $\lambda^{q^{n p}}$ converge, il faut et il suffit que $t$ puisse s'écrire

$$
t=\frac{r}{q^{p}-1}+\frac{u}{q^{v}}
$$

avec $r, u, v$ entiers et $v \geq 0$.

La limite $h(a)$ est alors constante et égale à $z=e^{2 \pi i s}$ avec $s=r /\left(q^{p}-1\right)$. Soit $h^{\prime}$ le PGCD des entiers $n>0$ pour lesquels il existe un mot $w=\left(w_{0} w_{1} \cdots w_{n}\right)$ de $u$ avec $w_{0}=w_{n}$. Pour que la constante $z$ soit un cobord, il faut et il suffit que $s h^{\prime}$ soit un entier. Soit $h$ le plus grand diviseur de $h^{\prime}$ premier avec $q$, et qui est appelé la hauteur de $\zeta$. Pour qu'un nombre $s$ puisse s'écrire sous la forme $s=r /\left(q^{p}-1\right)$ pour un certain $p$ et vérifie $h^{\prime} s \in \mathbb{Z}$, il faut et il suffit que $s h$ soit un entier. Finalement, $\lambda$ est une valeur propre si et seulement si $t$ peut s'écrire $t=(m / h)+\left(u / q^{v}\right)$. Remarquons que $h$ divise $q^{p}-1$, où $p$ est la période des initiales.

(2.3) A partir du théorème, on peut déterminer les valeurs propres rationnelles du système dynamique défini par une substitution de 2 lettres; ce résultat peut être extrait de l'article de Martin [3], mais sa forme précise a été proposée par le referee:

Proposition. Soit $\zeta$ une substitution de longueur non constante sur l'alphabet $\{0,1\}$, vérifiant les hypothèses du théorème. Soient $M$ la matrice de $\zeta, d$ le déterminant et $T$ la trace de $M$, et $t$ un rationnel. 


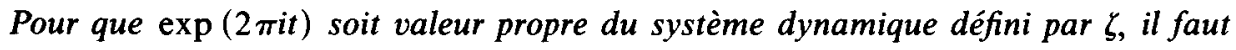
et il suffit que $t$ soit de la forme $t=(k / w)+\left(m / r^{n}\right)$, où $k, m, n$ sont des entiers, $n \geq 0, r=\operatorname{PGCD}(d, T)$ et où $w$ est l'entier défini par:

(i) les facteurs premiers de $w$ sont les nombres premiers qui divisent $|\zeta(0)|$ et $|\zeta(1)|$ mais pas $r$;

(ii) leurs exposants dans la décomposition de w sont les mêmes que dans la décomposition de $|\zeta(0)|-|\zeta(1)|$.

Pour $n>0$, soient $p_{n}=\left|\zeta^{n}(0)\right|$ et $q_{n}=\left|\zeta^{n}(1)\right|$; posons $p_{0}=q_{0}=1$.

Pour tout $n \geq 0$, on a alors

$$
\left(\begin{array}{l}
p_{n+1} \\
q_{n+1}
\end{array}\right)=\tilde{M}\left(\begin{array}{l}
p_{n} \\
q_{n}
\end{array}\right) .
$$

Soit $t=a / b$, où $a$ et $b$ sont deux entiers premiers entre eux. D'après le théorème, si $\exp (2 \pi i t)$ est valeur propre il existe $N>0$ tel que $b$ divise $p_{N}$ et $q_{N}$; réciproquement, si $b$ divise $p_{N}$ et $q_{N}$ pour un certain $N, b$ divise $p_{n}$ et $q_{n}$ pour tout $n \geq N$, et $\exp (2 \pi i t)$ est valeur propre. Il faut donc déterminer les nombres premiers $p$ et les entiers $k$ tels que $p^{k}$ divise $p_{N}$ et $q_{N}$ pour un certain $N$, ce qui est partiellement résolu par les lemmes 1 et 2 du $\S 3$ de [3]. On donne ici une démonstration rapide.

Pour tout $n \geq 0$, on a:

$$
\left.\begin{array}{l}
p_{n+1}=p_{1} p_{n}+M_{1,0}\left(q_{n}-p_{n}\right) \\
q_{n+1}=q_{1} q_{n}+M_{0,1}\left(p_{n}-q_{n}\right)
\end{array}\right\}
$$

et

$$
\left.\begin{array}{l}
p_{n+2}=T p_{n+1}-d p_{n} \\
q_{n+2}=T q_{n+1}-d q_{n}
\end{array}\right\}
$$

Soit $p$ un nombre premier. Si $p$ divise $r$, d'après (2) $p^{n}$ divise $p_{2 n}$ et $q_{2 n}$ pour tout $n>0$. Supposons que $p^{k}$ divise w. Alors $p$ divise $p_{1}$ et $q_{1}$, et $p^{k}$ divise $p_{1}-q_{1}$; d'après (1) et par récurrence, $p^{k}$ divise $p_{k}$ et $q_{k}$.

Réciproquement, supposons que $p^{k}$ divise $p_{N}$ et $q_{N}$, et que $p$ ne divise pas $r$. $p_{N+1}$ et $q_{N+1}$ sont divisibles par $p$. Si $p$ ne divisait pas $d$, d'après (2) et par récurrence descendante $p$ diviserait $p_{0}$ et $q_{0}$, ce qui est absurde; $p$ divise donc $d$, et ne divise donc pas $T$. D'après (2) et par récurrence descendante $p$ divise $p_{1}$ et $q_{1}$. Soit $l$ tel que $p_{1}-q_{1}$ soit divisible par $p^{1}$ mais pas par $p^{l+1}$. D'après (2) et par récurrence, $p^{1}$ divise $p_{n}-q_{n}$ pour tout $n$, et $p^{l+1}$ ne divise $p_{n}-q_{n}$ pour aucun $n$, donc $l \geq k$ et $p^{k}$ divise $w$. Finalement, pour qu'il existe $N$ tel que $p^{k}$ divise $p_{N}$ et $q_{N}$, il faut et il suffit qu'il existe $n$ tel que $p^{k}$ divise $r^{n} w$, ce qu'il fallait démontrer.

(2.4) On donne ici une méthode pour déterminer les valeurs $\lambda$ pour lesquelles les suites $\lambda^{\left|\zeta^{p n}(a)\right|}$ convergent.

Notons $A=\{0,1, \ldots, s-1\}$. Pour $x \in \mathbb{R}^{s}$, désignons par $\|x\|$ la distance de $x$ au point de $\mathbb{Z}^{s}$ le plus proche. On dira qu'une suite $\left(x_{n}\right)$ dans $\mathbb{R}^{s}$ converge vers $x$ modulo $\mathbb{Z}^{s}$ si $\left\|x_{n}-x\right\|$ tend vers 0 . Soit $M$ la matrice $(s, s)$ définie par:

$M_{i, j}$ est le nombre d'occurences de $i$ dans $\zeta(j)$. 
$M$ est appelée la matrice de $\zeta$. Notons encore $e$ le point de $\mathbb{R}^{s}$ dont toutes les coordonnées sont égales à 1 .

Pour tout $n>0$, le vecteur $\tilde{M}^{n} e$ a pour coordonnées $\left|\zeta^{n}(0)\right|,\left|\zeta^{n}(1)\right|, \ldots,\left|\zeta^{n}(s-1)\right|$. Soient enfin $t \in \mathbb{R}$ et $\lambda=e^{2 \pi i t}$. Alors:

Pour que, pour toute lettre $a$, la suite $\lambda^{\left|\zeta^{p n}(a)\right|}$ converge, il faut et il suffit que la suite $\left(t \tilde{M}^{p n} e\right)$ converge modulo $\mathbb{Z}^{s}$.

Le problème est alors réglé par le lemme suivant. Les parties (i) et (iii) du lemme seront utiles dans la suite

LemMe 1. Soient $P$ une matrice $(s, s)$ à coefficients entiers, et $x \in \mathbb{R}^{s}$.

(i) Si $\left(P^{n+1} x-P^{n} x\right)$ converge vers 0 modulo $\mathbb{Z}^{s}, P^{n} x$ converge modulo $\mathbb{Z}^{s}$.

(ii) Pour que $P^{n} x$ converge modulo $\mathbb{Z}^{s}$, il faut et il suffit que $x$ puisse s'écrire $x=x_{1}+x_{2}+x_{3}$ avec

$$
\begin{aligned}
& P^{n} x_{1} \rightarrow 0 \text { dans } \mathbb{R}^{s} ; \\
& P^{n} x_{2} \in \mathbb{Z}^{s} \text { pour tout } n \text { assez grand; } \\
& (P-I) x_{3} \in \mathbb{Z}^{s} .
\end{aligned}
$$

La suite $P^{n} x$ converge alors vers $x_{3}$ modulo $\mathbb{Z}^{s}$, et

(iii) la convergence est géométrique, c'est-à-dire qu'il existe $C>0$ et $r<1$ avec $\left\|P^{n} x-x_{3}\right\|<C r^{n}$ pour tout $n>0$.

Notons $y=P x-x$; pour tout $n>0$, soient $u_{n}$ le point de $\mathbb{Z}^{s}$ le plus proche de $P^{n} y$, et $v_{n}=P^{n} y-u_{n}$. La suite $v_{n}$ converge vers 0 ; pour tout $n, u_{n+1}-P u_{n}=P v_{n}-v_{n+1}$, donc la suite $u_{n+1}-P u_{n}$ converge vers 0 , et, comme cette suite est à valeurs dans $\mathbb{Z}^{s}$, elle est nulle à partir d'un certain rang. Il existe ainsi $m>0$ tel que $u_{m+n}=P^{n} u_{m}$ pour tout $n \geq 0$. Comme l'image des opérateurs $P^{s}$ et $P^{m+s}$ est la même, il existe $y_{2} \in \mathbb{R}^{s}$ tel que $P^{m+s} y_{2}=P^{s} u_{m}$, et ainsi $u_{n}=P^{n} y_{2}$ pour tout $n \geq q=m+s$.

Soit $y_{1}=y-y_{2}$. Pour $n \geq q, P^{n} y_{1}=v_{n}$, donc la suite $P^{n} y_{1}$ converge vers $0 ; y_{1}$ appartient donc au sous-espace de $\mathbb{R}^{s}$ algébriquement associé aux valeurs propres de $P$ de module strictement infèrieur à 1 ; la restriction de $P-I$ à ce sous-espace est un isomorphisme, et il existe $x_{1}$ dans ce sous-espace tel que $y_{1}=P x_{1}-x_{1}$; la suite $P^{n} x_{1}$ converge alors géométriquement vers 0 .

Soit $z=x-x_{1}$. On a $P z-z=y_{2}$, et $P^{q}(P-I) z=u_{q} \in \mathbb{Z}^{s}$. Posons $x_{2}=z-P^{q} z$ et $x_{3}=P^{q} z$. On a bien $P^{q} x_{2}=-\left(I+P+\cdots+P^{q-1}\right) u_{q} \in \mathbb{Z}^{s},(P-I) x_{3}=u_{q} \in \mathbb{Z}^{s}$ et $x=$ $x_{1}+x_{2}+x_{3}$. Enfin, pour $n \geq q$,

$$
\left\|P^{n} x-x_{3}\right\|=\left\|P^{n}\left(x_{1}+x_{2}\right)\right\|=\left\|P^{n} x_{1}\right\|
$$

et cette suite converge vers 0 géométriquement.

\section{Topologie de $X$}

Considérée comme une application de $A^{\mathbb{N}}$ dans lui-même, $\zeta$ est continue. D'autre part, $\zeta(u)=u$ et pour tout $n>0$,

$$
\zeta\left(T^{n} u\right)=T^{\mid \zeta(u[0, n[) \mid} u,
$$

donc l'orbite fermée $X$ de $u$ est stable par $\zeta$; on étudie maintenant les propriétés de l'application continue $\zeta$ de $X$ dans lui-même. 
(3.1) LeMme 2. (i) Soit $\left(n_{i}\right)$ une suite d'entiers positifs telle que ( $\left.T^{n_{i}} u\right)$ converge vers un point $x \in X$. Pour que $x$ appartienne à $\zeta(X)$, il faut et il suffit que, pour tout $i$ assez grand, $n_{i}$ appartienne à l'ensemble E défini en (1.2). En particulier, $T^{r} u$ appartient d̀ $\zeta(X)$ si et seulement si $r \in E$.

(ii) $\zeta(X)$ est une partie ouverte et fermée de $X$.

Supposons d'abord que pour tout $i$ assez grand $n_{i} \in E$, et que la suite ( $T^{n}{ } u$ ) converge vers $x$. Pour $i$ assez grand, il existe $m_{i}$ tel que $n_{i}=\mid \zeta\left(u\left[0, m_{i}[) \mid\right.\right.$; soit $y$ un point adhérant à la suite $\left(T^{m_{i}} u\right)$; alors $\zeta\left(T^{m_{i}} u\right)=T^{n_{i}} u$ pour $i$ assez grand, d'après (*), donc $\zeta(y)=x$, et finalement $x \in \zeta(X)$.

Réciproquement, soient $\left(n_{i}\right)$ une suite d'entiers telle que $\left(T^{n_{i}} u\right)$ converge vers le point $x \in \zeta(X), y \in X$ tel que $\zeta(y)=x$, et $\left(m_{i}\right)$ une suite d'entiers telle que $\left(T^{m_{i}} u\right)$ converge vers $y$. Pour tout $i$, soit $r_{i}=\mid \zeta\left(u\left[0, m_{i}[) \mid ; r_{i}\right.\right.$ appartient à $E$, et $T^{r_{i}} u=\zeta\left(T^{m_{i}} u\right)$. La suite $\left(T^{r_{i}} u\right)$ converge vers la même limite $x$ que la suite $\left(T^{n_{i}} u\right) ; k$ étant l'indice de reconnaissabilité de $\zeta$ introduit en (1.2), pour tout $i$ assez grand $u\left[r_{i}, r_{i+k}\left[=u\left[n_{i}, n_{i+k}\left[\right.\right.\right.\right.$, donc $n_{i}$ appartient à $E$. Ceci prouve la partie (i) du lemme, et la partie (ii) s'en déduit immédiatement.

(3.2) Lemme 3. (i) Soient $x \in X$ et $p>0$. Pour que $T^{p} \zeta(x)$ appartienne à $\zeta(X)$, il faut et il suffit qu'il existe un entier $r>0$ tel que $p=\mid \zeta\left(x\left[0, r[) \mid\right.\right.$, et alors $T^{p} \zeta(x)=$ $\zeta\left(T^{r} x\right)$.

(ii) $\zeta$ est un homéomorphisme de $X$ sur $\zeta(X)$.

Pour démontrer la partie (i) du lemme, il suffit de prouver que le plus petit entier $p>0$ tel que $T^{p} \zeta(x) \in \zeta(X)$ est $p=\left|\zeta\left(x_{0}\right)\right|$.

On a bien $T^{p} \zeta(x)=\zeta(T x) \in \zeta(X)$. Inversement, soit $0<q<p$, et supposons que $T^{q} \zeta(x) \in \zeta(X)$. Soit $\left(n_{i}\right)$ une suite d'entiers telle que $\left(T^{n_{i}} u\right)$ converge vers $x$, et $m_{i}=\mid \zeta\left(u\left(0, n_{i}[) \mid ;\right.\right.$ pour tout $i, m_{i} \in E$. La suite $\left(T^{m_{i}+q} u\right)$ converge vers le point $T^{q} \zeta(x)$ de $\zeta(X)$, donc $m_{i}+q$ appartient à $E$ pour tout $i$ assez grand d'après le lemme 2(i). Or, pour $i$ assez grand, $u_{n_{i}}=x_{0}$, donc l'entier suivant $m_{i}$ dans $E$ est $m_{i}+p$, d'où une contradiction.

Pour démontrer la partie (ii) du lemme, il suffit de prouver que $\zeta$ est injective. Soient $x, y \in X$ avec $\zeta(x)=\zeta(y)=z$. D'après (i), le plus petit entier $p>0$ tel que $T^{p} z \in \zeta(X)$ est égal à la fois à $\left|\zeta\left(x_{0}\right)\right|$ et à $\left|\zeta\left(y_{0}\right)\right|$. Alors $\zeta\left(x_{0}\right)=z\left[0, p\left[=\zeta\left(y_{0}\right)\right.\right.$, et, par injectivité de $\zeta$ sur les lettres, $x_{0}=y_{0}$. Par récurrence, $x_{n}=y_{n}$ pour tout $n$, et $x=y$.

(3.3) Soit $n>0$; la substitution $\zeta^{n}$ est primitive, et elle définit la même suite $u$, et le même système dynamique que la substitution $\zeta$. De plus, elle possède les mêmes propriétés que $\zeta$ :

LeMme 4. Pour tout $n>0$, la substitution $\zeta^{n}$ est reconnaissable, et les lemmes 2 et 3 sont valables pour elle; dans la partie (i) du lemme 2, il faut bien sûr remplacer l'ensemble $E$ par l'ensemble $E_{n}$ défini par:

$$
E_{n}=\{0\} \cup\left\{\mid \zeta^{n}(u[0, p[) \mid ; p>0\} .\right.
$$

Par récurrence, $\zeta^{n}$ est un homéomorphisme, et $\zeta^{n}(X)$ est une partie ouverte de $X$.

Soit $n>0$. Supposons que $\zeta^{n}$ est reconnaissable, et soit $k_{n}$ son indice de reconnaissabilité. Montrons que $\zeta^{n+1}$ est reconnaissable. Comme $\zeta^{n}$ est un homéomor- 
phisme, il existe $k_{n+1} \geq k_{n}$ tel que, pour $x, y \in X$,

$$
\zeta^{n}(x)\left[0, k_{n+1}\left[=\zeta^{n}(y)\left[0, k_{n+1}[\right.\right.\right.
$$

entraîne $x\left[0, k\left[=y\left[0, k\left[\right.\right.\right.\right.$, où $k=k_{1}$ est l'indice de reconnaissabilité de $\zeta$. Soient $p$ et $q$ deux entiers tels que $p \in E_{n+1}$ et que $u\left[p, p+k_{n+1}\left[=u\left[q, q+k_{n+1}[\right.\right.\right.$. Il existe $r>0$ tel que $T^{p} u=\zeta^{n+1}\left(T^{r} u\right)$; d'autre part, comme $k_{n+1} \geq k_{n}, q \in E_{n}$, et il existe $m$ tel que $T^{q} u=\zeta^{n}\left(T^{m} u\right)$. Alors, par définition de $k_{n+1},\left(\zeta\left(T^{r} u\right)\right)\left[0, k\left[=\left(T^{m} u\right)[0, k[\right.\right.$, donc $m \in E$, et il existe $l$ tel que $T^{m} u=\zeta\left(T^{l} u\right)$. Finalement, $T^{q} u=\zeta^{n+1}\left(T^{l} u\right)$, et $q \in E_{n+1}$.

Ainsi, $\zeta^{n}$ est reconnaissable pour tout $n$, et les propriétés (i) du lemme 2 et du lemme 3 s'en déduisent.

Il n'est pas clair que la substitution $\zeta^{n}$ est injective sur les lettres pour $n>1$; cependant, on n'utilisera plus cette propriété de $\zeta$ dans la suite, mais seulement les conclusions des lemmes précédents, et on pourra donc remplacer $\zeta$ par un itéré $\zeta^{n}$ chaque fois que ce sera utile.

\section{Fonctions propres continues}

On commence ici la démonstration du théorème, en montrant qu'un complexe $\lambda$ vérifiant les hypothèses de convergence du théorème est une valeur propre correspondant à une fonction propre continue de $(X, T)$. On remplace la substitution $\zeta$ par son itérée $\zeta^{p}$.

(4.1) Un lemme de convergence uniforme. Soit $\lambda=e^{2 \pi i t}$ un nombre complexe de module 1 tel que, pour toute lettre $a \in A$, la limite $h(a)=\lim _{n \rightarrow \infty} \lambda^{\left|\zeta^{\prime \prime}(a)\right|}$ existe. Avec les notations du $\S 2$, on a vu qu'alors la suite $\left(t \tilde{M}^{n} e\right)$ converge modulo $\mathbb{Z}^{s}$, et, d'après le lemme 1 , cette convergence est géométrique: il existe $C<0$ et $0<r<1$ tels que, pour toute lettre $a$ et tout $n>0$,

$$
\left|\lambda^{\left|\zeta^{n}(a)\right|}-h(a)\right| \leq C r^{n} .
$$

Pour $w=\left(w_{0} w_{1} \cdots w_{p}\right) \in A^{*}$, notons $h(w)=h\left(w_{0}\right) h\left(w_{1}\right) h\left(w_{2}\right) \cdots h\left(w_{p}\right)$. Pour tout mot $w$ de $u$, on a alors

$$
\left|\lambda^{\left|\zeta^{n}(w)\right|}-h(w)\right| \leq|w| C r^{n}
$$

Lemma 5. Soit $\lambda$ un complexe de module 1 vérifiant la condition de convergence précédente, et $h$ comme ci-dessus. Il existe $B>0$ et $0<r<1$ tels que, pour tout $m>0$

$$
\mid \lambda^{\mid \zeta^{n}(u[0, m[) \mid}-h\left(u \left[0, m[) \mid \leq B r^{n} .\right.\right.
$$

Soit $m>0$. Pour tout $i>0$, soit $p_{i}$ le plus grand entier $\leq m$ et appartenant à $E_{i}$ (les ensembles $E_{i}$ ont été définis en (3.3)). La suite $\left(p_{i}\right)$ est décroissante, et $p_{i}=0$ pour tout $i$ supérieur ou égal à un certain entier $q$.

Soit $0<i<q$. Si $p_{i+1}<p_{i}, u\left[p_{i+1}, p_{i}\right.$ [ peut s'écrire $\zeta^{i}\left(w_{i}\right)$, où $w_{i}$ est un mot de $u$ avec $\left|w_{i}\right|<S=\operatorname{Sup}\{|\zeta(a)| ; a \in A\}$. Si $p_{i+1}=p_{i}$, on pose $w_{i}=\varnothing$. De même, si $p_{1}<m$, on pose $w_{0}=u\left[p_{1}, m\left[\right.\right.$ et $w_{0}=\varnothing$ si $p_{1}=m$; on a encore $\left|w_{0}\right|<S$. En posant $\zeta(\varnothing)=\varnothing$ et $h(\varnothing)=1$, on a alors $u\left[0, m\left[=\zeta^{q-1}\left(w_{q-1}\right) \cdots \zeta^{1}\left(w_{1}\right) w_{0}\right.\right.$ d'où

$$
\lambda^{\mid \zeta^{n}(u[0, m[) \mid}=\lambda^{\left|\zeta^{n+q-1}\left(w_{q-1}\right)\right|} \cdots \lambda^{\left|\zeta^{n}\left(w_{0}\right)\right|}
$$


et

$$
h\left(u \left[0, m[)=h\left(\zeta^{q-1}\left(w_{q-1}\right)\right) \cdots h\left(w_{0}\right) .\right.\right.
$$

Or, par définition de $h, h(\zeta(w))=h(w)$ pour tout $w \in A^{*}$, donc

$$
h\left(u \left[0, m[)=h\left(w_{q-1}\right) \cdots h\left(w_{0}\right) .\right.\right.
$$

D'autre part, pour $0 \leq i<q$

$$
\left|\lambda^{\left|\zeta^{n+i}\left(w_{i}\right)\right|}-h\left(w_{i}\right)\right| \leq C r^{n+i} S .
$$

Finalement,

$$
\mid \lambda^{\mid \zeta^{n}(u[0, m[) \mid}-h\left(u \left[0, m[) \mid \leq \sum_{i} C r^{n+i} S \leq C r^{n} S /(1-r),\right.\right.
$$

ce qui est le résultat cherché.

(4.2) Proposition 1. Soit $\lambda$ un complexe de module 1 tel que, pour toute lettre a, la limite $h(a)=\lim _{n \rightarrow \infty} \lambda^{\left|\zeta^{n}(a)\right|}$ existe, et que l'application $h$ soit un cobord de $\zeta$. Alors $\lambda$ est une valeur propre du système dynamique $(X, T)$, correspondant à une fonction propre $g$ continue.

Soit $f: A \mapsto T$ une fonction associée au cobord $h$, c'est-à-dire telle que, pour tout mot $(a b)$ de 2 lettres de $u$, on ait $f(b)=f(a) h(a)$. Soit $n>0$. Pour tout $x \in X$, soit $r_{n}(x)$ le plus petit entier $\geq 0$ tel que $T^{r_{n}(x)} x$ appartienne à $\zeta^{n}(X)$. Comme $\zeta^{n}(X)$ est ouvert et fermé dans $X$, la fonction $r_{n}$ est continue sur $X$.

Pour $a \in A$, notons $[a]$ le cylindre $\left\{x \in X ; x_{0}=a\right\}$. Quand $a$ parcourt $A$, les ensembles $[a]$ forment une partition ouverte et fermée de $X$, et les ensembles $\zeta^{n}([a])$ forment une partition ouverte et fermée de $\zeta^{n}(X)$. Posons:

$$
g_{n}(x)=\lambda^{-r_{n}(x)} f(a) \quad \text { si } T^{r_{n}(x)} x \in \zeta^{n}([a])
$$

La fonction $g_{n}$ est continue sur $X$. Soit $p>0$, et $x=T^{p} u . T^{r_{n}(x)+p} u$ appartient à $\zeta^{n}(X)$; d'après lemme $2, r_{n}(x)+p$ appartient à $E_{n}$ et il existe $q \geq 0$ avec

$$
T^{r_{n}(x)+p} u=\zeta^{n}\left(T^{q} u\right) \quad \text { et } \quad r_{n}(x)+p=\mid \zeta^{n}(u[0, q[) \mid \text {. }
$$

Alors $T^{r_{n}(x)} x \in \zeta^{n}\left(\left[u_{q}\right]\right)$ donc

$$
g_{n}(x)=f\left(u_{q}\right) \lambda^{-r_{n}(x)}=f\left(u_{0}\right) h\left(u \left[0, q[) \lambda^{-r_{n}(x)}\right.\right.
$$

D'autre part, $g_{n}(u)=f\left(u_{0}\right)$, d'où:

$$
\left|g_{n}\left(T^{p} u\right)-\lambda^{p} g_{n}(u)\right|=\mid h\left(u \left[0, q[)-\lambda^{p+r_{n}(x)}|=| h\left(u \left[0, q[)-\lambda^{\mid \zeta^{n}(u[0, q[) \mid} \mid \leq B r^{n},\right.\right.\right.\right.
$$

d'après le lemme 5 .

Soient $n$ et $m$ deux entiers. Pour tout $p \geq 0,\left|g_{n}\left(T^{p} u\right)-g_{m}\left(T^{p} u\right)\right| \leq B\left(r^{n}+r^{m}\right)$; par densité de la suite $\left(T^{p} u\right)$ dans $X$, la suite $\left(g_{n}\right)$ converge uniformément vers une fonction continue $g$ sur $X$. Pour tout $p, g\left(T^{p} u\right)=\lambda^{p} g(u)$ et, par densité, $g(T x)=$ $\lambda g(x)$ pour tout $x \in X$, ce qu'il fallait démontrer.

\section{Fonctions propres mesurables}

On démontre ici la deuxième partie du théorème, en prouvant que toute valeur propre du système $(X, T, \mu)$ vérifie les conditions imposées.

Fixons d'abord quelques notations. La matrice $M$ de $\zeta$ est primitive car la substitution $\zeta$ est primitive. On notera $\theta$ la valeur propre de Perron-Frobenius de 
$M$. On sait qu'il existe une constante $P>0$ telle que $P \theta^{n}<\left|\zeta^{n}(a)\right|$ pour toute lettre $a$ et tout $n>0$.

Pour $w=\left(w_{0} \cdots w_{p}\right) \in A^{*}$, on notera $[w]$ le cylindre défini par $w$, c'est-à-dire l'ensemble des $x \in X$ tel que $x[0, p[=w$.

(5.1) Propriétés métriques. On a vu qu'il existe une partie $D$ de $X$, dénombrable et donc négligeable, telle que $T$ soit un homéomorphisme de $X \backslash D$; en particulier, $T$ est un isomorphisme au sens métrique.

Pour $n>0$, notons $T_{n}$ la transformation induite par $T \operatorname{sur} \zeta^{n}(X)$. D'après le lemme 3 , appliqué à $\zeta^{n}$,

$$
T_{n} \zeta^{n}(x)=T^{\left|\zeta^{n}\left(x_{0}\right)\right|} \zeta^{n}(x)=\zeta^{n}(T x)
$$

pour tout $x \in X . \zeta^{n}$ est donc un isomorphisme du système $(X, T) \operatorname{sur}\left(\zeta^{n}(X), T_{n}\right)$ et, en particulier, ce dernier est uniquement ergodique. La restriction de $\mu$ à $\zeta^{n}(X)$, non nulle par minimalité, est invariante par $T_{n}$, ainsi que la mesure image $\zeta^{n}(\mu)$ : ces deux mesures sont proportionnelles et il est facile de voir (cf. [4]) que le coefficient de proportionnalité est $\theta$. Ainsi, pour toute partie $Y$ mesurable de $X, \mu\left(\zeta^{n}(Y)\right)=\theta^{-n} \mu(Y)$.

(5.2) Pour $n>0$, notons $\Sigma_{n}$ l'ensemble des couples $(a, p)$, où $a$ est une lettre et $p$ un entier avec $0 \leq p<\left|\zeta^{n}(a)\right|$. Soit $\mathscr{P}_{n}$ la famille des ensembles $T^{p} \zeta^{n}([a])$ où $(a, p)$ parcourt $\Sigma_{n}$.

$\mathscr{P}_{n}$ est un recourvrement de $X$ car la réunion de ces ensembles est un fermé qui contient $T^{k} u$ pour tout $k \geq 0$. Soient $(a, p)$ et $(b, q)$ dans $\Sigma_{n}$, avec $p \geq q$, et supposons que

$$
T^{p} \zeta^{n}([a]) \cap T^{q} \zeta^{n}([b]) \cap(X \backslash D) \neq \varnothing .
$$

Alors, $T^{p-a} \zeta^{n}([a]) \cap \zeta^{n}([b]) \neq \varnothing$; soit $x \in X$ vec $x_{0}=a$ et $T^{p-q} \zeta^{n}(x) \in \zeta^{n}([b])$. Le plus petit entier $k>0$ tel que $T^{k} \zeta^{n}(x)$ appartient à $\zeta^{n}(X)$ est, d'après le lemme 3, $k=\left|\zeta^{n}(a)\right|$, donc $k>p-q$ et $p=q$. $\zeta^{n}$ étant injective, $x \in[b]$, d'où $a=b$. Ainsi, l'intersection de 2 éléments de $\mathscr{P}_{n}$ correspondant à des éléments distincts de $\Sigma_{n}$ est incluse dans l'ensemble négligeable $D$ et $\mathscr{P}_{n}$ est une partition de $X$ au sens métrique.

Pour toute fonction $g \in L^{2}(\mu)$, notons $\mathscr{E}_{n} g$ l'espérance conditionnelle de $g$ sachant la tribu $\mathscr{B}_{n}$ engendrée par $\mathscr{P}_{n}$.

LEMME 6. Pour toute fonction $g \in L^{1}(\mu)$, la suite $\left(\mathscr{C}_{n} g\right)$ converge vers $g$ dans $L^{1}(\mu)$.

On peut se restreindre à montrer cette propriété dans le cas où $g$ est la fonction indicatrice d'un cylindre [ $w]$. Notons $k=|w|$.

Soient $n>0,(a, p) \in \Sigma_{n}$ et $Y=T^{p} \zeta^{n}([a])$. Si $p \leq\left|\zeta^{n}(a)\right|-k$ et si $\left(\zeta^{n}(a)\right)[p, p+k[$ $=w, Y$ est inclus dans $[w]$ et $\mathscr{E}_{n} g=g$ sur $Y ; p \leq\left|\zeta^{n}(a)\right|-k$ et $\left(\zeta^{n}(a)\right)[p, p+k[$ $\neq w, Y \cap[w]=\varnothing$ et $\mathscr{E}_{n} g=0=g$ sur $Y$. Les seuls ensembles de $\mathscr{P}_{n}$ où $\mathscr{E}_{n} g$ peut êntre différente de $g$ correspondent donc à des éléments $(a, p)$ de $\Sigma_{n}$ tels que $\left|\zeta^{n}(a)\right|-k<$ $p<\left|\zeta^{n}(a)\right| ; s$ étant le nombre de lettres de $A$, il y a au plus $s(k-1)$ ensembles de ce type; la mesure de chacun d'eux est majorée par $\mu\left(\zeta^{n}(X)\right)=\theta^{-n}$, et finalement

$$
\left\|g-\mathscr{E}_{n} g\right\|_{L^{1}(\mu)} \leq(k-1) s \theta^{-n}
$$


(5.3) Proposition 2. Soit g une fonction propre du système $(X, T, \mu)$, correspondant à la valeur propre $\lambda$, et soit p la période des initiales définite en (2.1). Pour toute lettre $a$, la limite

$$
h(a)=\lim _{n \rightarrow \infty} \lambda^{\left|\zeta^{p n}(a)\right|}
$$

existe, et $h$ est un cobord de $\zeta$.

Quitte à remplacer $\zeta$ par $\zeta^{p}$, on peut supposer que la période des initiales de $\zeta$ est 1. $g$ a un module constant et on peut supposer que $|g|=1$ partout.

Pour $n>0$, notons $g_{n}=\mathscr{E}_{n} g$; pour toute lettre $a, g_{n}$ est constante sur $\zeta^{n}([a])$; notons $d_{n}(a)$ cette constante.

Soit $a$ une lettre. Pour $n>0$ et $0 \leq p<\left|\zeta^{n}(a)\right|, g_{n}$ est constante et égale à $\lambda^{p} d_{n}(a)$ $\operatorname{sur} T^{p} \zeta^{n}([a])$; alors, pour tout $n>0$,

$$
\begin{aligned}
\int\left|g-g_{n}\right| d \mu & =\sum_{0 \leq p<\left|\zeta^{n}(a)\right|} \int_{T^{p} \zeta^{n}([a])}\left|g-g_{n}\right| d \mu \\
& =\left|\zeta^{n}(a)\right| \int_{\zeta^{n}([a])}\left|g-d_{n}(a)\right| d \mu \geq P \theta^{n} \int_{\zeta^{n}([a])}\left|g-d_{n}(a)\right| d \mu .
\end{aligned}
$$

Comme la suite $\left(g_{n}\right)$ converge vers $g$ dans $L^{1}(\mu)$, on obtient:

$$
\text { Pour tout } a \in A, \theta^{n} \int_{\zeta^{n}([a])}\left|g-d_{n}(a)\right| d \mu \text { tend vers } 0 .
$$

En particulier, comme $|g|=1$, que $\left|d_{n}(a)\right| \leq 1$ et que $\mu\left(\zeta^{n}([a])\right)=\theta^{-n} \mu([a])$, on a:

$$
\text { Pour tout } a \in A, \lim _{n \rightarrow \infty}\left|d_{n}(a)\right|=1 \text {. }
$$

Soient $a$ une lettre et $b$ la première lettre de $\zeta(a) . \zeta([a])$ est inclus dans $[b]$, donc $\zeta^{n+1}([a]) \subset \zeta^{n}([b])$ pour tout $n>0$. Alors, pour tout $n>0$,

$$
\begin{aligned}
\mu\left([a]\left|d_{n+1}(a)-d_{n}(b)\right|\right. & =\theta^{n+1} \int_{\zeta^{n+1}([a])}\left|g_{n}-g_{n+1}\right| d \mu \\
& \leq \theta^{n+1}\left(\int_{\zeta^{n+1}([a])}\left|g_{n+1}-g\right| d \mu+\int_{\zeta^{n}([b])}\left|g_{n}-g\right| d \mu\right)
\end{aligned}
$$

qui tend vers 0 d'après (1). D'après (2), on obtient:

Si $a \in A$, et si $b$ est la première lettre de $\zeta(a)$,

$$
\lim _{n \rightarrow \infty} d_{n+1}(a) / d_{n}(b)=1 .
$$

Soit $a$ une lettre. Posons $a_{0}=a$ et, pour tout $i \geq 0$, soit $a_{i+1}$ la première lettre de $\zeta\left(a_{i}\right)$. Comme la période des initiales de $\zeta$ est 1 , il existe $k$ tel que $a_{k}=a_{k+1}$. D'après (3)

$$
\lim _{n \rightarrow \infty} d_{n+k}(a) / d_{n}\left(a_{k}\right)=1 \text { et } \quad \lim _{n \rightarrow \infty} d_{n+k+1}(a) / d_{n}\left(a_{k+1}\right)=1 .
$$

Par conséquent,

$$
\text { Pour tout } a \in A, \lim _{n \rightarrow \infty} d_{n+1}(a) / d_{n}(a)=1 \text {. }
$$

Soient $a$ et $b$ deux lettres telles que $w=(a b)$ soit un mot de $u$. Alors, $[w] \subset[a]$, et $T[w] \subset[b]$. Soit $n>0$; notons $k=\left|\zeta^{n}(a)\right|$. On a $\zeta^{n}([w]) \subset \zeta^{n}([a])$ et $T^{k} \zeta^{n}([w]) \subset$ 
$\zeta^{n}([b])$. D'une part,

$$
\begin{aligned}
\int_{\zeta^{n}([w])}\left|g-\lambda^{-k} d_{n}(b)\right| d \mu & =\int_{T^{k} \zeta^{n}([w])}\left|g-d_{n}(b)\right| d \mu \\
& \leq \int_{\zeta^{n}([b])}\left|g-d_{n}(b)\right| d \mu .
\end{aligned}
$$

D'autre part,

$$
\int_{\zeta^{n}([w])}\left|g-d_{n}(a)\right| d \mu \leq \int_{\zeta^{n}([a])}\left|g-d_{n}(a)\right| d \mu .
$$

Par minimalité, $\mu([w]) \neq 0 ; \mu\left(\zeta^{n}([w])\right)=\theta^{-n} \mu([w])$, donc d'après (1),

$$
\lim _{n \rightarrow \infty}\left|d_{n}(b)-\lambda^{\left|\zeta^{n}(a)\right|} d_{n}(a)\right|=0 \text {. }
$$

Finalement, d'après (2), si $a$ et $b$ sont deux lettres telles que ( $a b)$ soit un mot de $u$,

$$
\lim _{n \rightarrow \infty} \lambda^{\left|\zeta^{n}(a)\right|} d_{n}(a) / d_{n}(b)=1 .
$$

Pour toute lettre $a$, il existe une lettre $b$ telle que $(a b)$ soit un fot de $u$. Donc, d'après (5) et (4),

$$
\text { Pour toute lettre } a, \lambda^{\left|\zeta^{n+1}(a)\right|-\left|\zeta^{n}(a)\right|}=1 .
$$

Soit $t \in \mathbb{R}$ avec $\lambda=e^{2 \pi i t} . M$ désignant toujours la matrice de $\zeta$, et $e$ le point de $\mathbb{R}^{s}$ dont toutes les coordonnées sont égales à 1 , la propriété (6) signifie que la suite $\left(\tilde{M}^{n+1} t e-\tilde{M}^{n} t e\right)$ converge modulo $\mathbb{Z}^{s} ;$ d'après le lemme 1 , la suite $\left(\tilde{M}^{n} t e\right)$ converge modulo $\mathbb{Z}^{s}$, donc:

$$
\text { Pour toute lettre } a \text {, la limite } h(a)=\lim _{n \rightarrow \infty} \lambda^{\left|\zeta^{n}(a)\right|} \text { existe. }
$$

Il reste à prouver que $h$ est un cobord de $\zeta$. D'après (5) et (7), la limite $\lim _{n \rightarrow \infty} d_{n}(a) / d_{n}(b)$ existe si $(a b)$ est un mot de $u$; cette limite existe donc pour tout couple $(a b)$ de lettres. Pour toute lettre $a$, posons $f(a)=\lim _{n \rightarrow \infty} d_{n}(a) / d_{n}(0)$. D'après (5), si $a$ et $b$ sont deux lettres telles que $(a b)$ soit un mot de $u, h(a) f(a)=$ $f(b)$, ce qui prouve que $h$ est un cobord de $\zeta$, et qui achève la démonstration de la proposition 2 . Le théorème se déduit alors immédiatement de celle-ci et de la proposition 1 .

\section{Exemples}

(6.1) Le système dynamique défini par une substitution peut être faiblement mélangeant, comme le montre l'exemple suivant.

Soit $\zeta$ la substitution définie sur l'alphabet $A=\{0,1\}$ par:

$$
\begin{aligned}
& \zeta(0)=(01010) \\
& \zeta(1)=(011) .
\end{aligned}
$$

Cette substitution vérifie les hypothèses du théorème (son indice de reconnaissabilité est 4), sa période des initiales est 1 , et son seul cobord est la constante 1 . D'autre part, pour tout $n>0,2\left|\zeta^{n}(1)\right|-\left|\zeta^{n}(0)\right|=1$. D'après le théorème, le seule valeur propre du système défini par cette substitution est 1 , et ce système est faiblement mélangeant. 
(6.2) Des considérations algébriques permettent parfois de préciser le résultat du théorème. On donne ici 2 exemples, sans démonstration, pour illustrer la diversité des situations possibles. Désormais, $\zeta$ est une substitution sur l'alphabet $A=\{0,1, \ldots s-1\}$, vérifiant les hypothèses du théorème, et n'admettant comme cobord que la constante 1 . Comme au (2.3), $M$ désigne la matrice de $\zeta ; \theta$ désigne la valeur propre de Perron-Frobenius de $M$.

Supposons d'abord que $\theta$ est la seule valeur propre de $M$ de module $\geq 1$. Il existe alors, pour toute lettre $a \in A$, un réel $q_{a}>0$ tel que

$$
\text { Pour tous } a, b \in A, \lim _{n \rightarrow+\infty} M_{a b}^{n}-\theta^{n} \mu[a] q_{b}=0 .
$$

On en déduit que, pour toute lettre $a, \exp (2 \pi i \mu[a])$ est une valeur propre du système dynamique défini par $\zeta$.

Si de plus le déterminant de $M$ est non-nul, les réels $\mu[a]$ sont irrationnels, et l'ensemble $\{\mu[a] ; a>0\}$ est rationnellement indépendant. Enfin, si le déterminant de $M$ est \pm 1 , le groupe des valeurs propres du système dynamique défini par $\zeta$ est engendré par $\{\exp (2 \pi i \mu[a]) ; a \in A\}$, et ces valeurs propres sont toutes irrationnelles.

(6.3) A l'opposé, dans certains cas les valeurs propres sont toutes rationnelles.

Soit $P(X)$ le polynôme minimal de $\theta$, c'est à dire le polynôme unitaire à coefficients entiers de plus petit degré qui s'annule en $\theta$. Supposons que le nombre de racines de $P(X)$ de module $\geq 1$ soit strictement supèrieur au nombre de racines de $P(X)$ de module $<1$. Cette hypothèse est vérifiée en particulier si $\theta$ est un entier, et donc si la substitution $\zeta$ est de longueur constante. Alors toutes les valeurs propres du système dynamique défini par $\zeta$ sont rationnelles: pour que $\lambda=\exp (2 \pi i t)$ soit valeur propre, il faut et il suffit qu'il existe $n$ tel que:

pour toute lettre $a, t\left[\zeta^{n}(a)\right]$ est un entier.

\section{RÉFÉRENCES}

[1] F. M. Dekking. The spectrum of dynamical systems arising from substitutions of constant length. Z. Wahr. Verw. Geb. 41 (1978), 221-239.

[2] W. H. Gottschalk. Substitution minimal sets. Trans. Amer. Math. Soc. 109 (1963), 467-491.

[3] J. C. Martin. Minimal flows arising from substitutions of non-constant length. Math. Systems Th. 7 (1973), 73-82.

[4] M. Queffelec. Contribution à l'étude spectrale des suites arithmétiques. Thèse, Université de ParisNord (1984). 